\title{
Sensitivity of regions of irregular and chaotic vibrations of an asymmetric rotor supported on journal bearings to structural parameters
}

Received: 31 December 2014 / Revised: 17 April 2015 / Published online: 30 December 2015

(C) The Author(s) 2015. This article is published with open access at Springerlink.com

\begin{abstract}
Rotating elements supported on journal bearings are widely encountered structures in engineering practice. Most commonly, these are asymmetrically manufactured and loaded rigid rotors transmitting torque and carrying transverse as well as axial forces. Nowadays, despite high operational demands and high rotational velocities, such systems are still expected to exhibit stable working, even in the presence of small assembly deviations, light unbalance or external disturbances. The surrounding environment of a rotating machine may interact with it by kinematic excitation from vibrating foundation. This, in turn, may lead to hazardous response and the onset of irregular and chaotic motion of the rotor. The subject of the study is to find and analyze regions of the occurrence of such vibrations in the system of a rigid rotor supported in journal bearings. The bearings themselves are assumed to be non-perfectly mounted in the housing, i.e., their sleeves are inserted in rings possessing some viscoelastic properties. These properties are treated as variable parameters, and the aim is to move the regions of irregular and chaotic vibration outside the operational regime (angular velocity). The adjustability of the viscoelastic parameters may be realized by incorporation of smart materials such as piezoelectric or magnetorheological ones. The considered system is an asymmetric rigid rotor supported on two journal bearings subject to a steady kinematic excitation. The system is described by eight coupled nonlinear ordinary differential equations of motion. Results of the examinations prove that by selecting an appropriate magnitude of damping and stiffness of the bearing mountings, it is possible to enlarge the region of stable operation of the rotating system and thus secure its safety. This, however, does not mean the elimination of chaotic response at all, but only a shift of it outside the range of operational rotation speed.
\end{abstract}

\section{Introduction}

Journal bearing systems keep their leadership among the most popular supporting elements being applied to machines practically of any purpose and destination for decades. They are reliable supports of turbines, generators, and engines. However, expectations of the industry become more and more rigorous and demanding. The bearings have to work stably in regions of continuously higher rotation speeds, and any appearances of sudden jumps of vibration amplitude are inadmissible. In the literature, one comes across a variety of concepts toward novel solutions that follow engineers' and researchers' visions of what can be done about stability of journal bearings. Recently, much attention has been paid to passive, semi-active and active methods of stabilization and reduction of vibration of rotating shafts. These methods often incorporate smart materials and sophisticated control algorithms to accomplish the task. For instance, Bonneau et al. [5] employed an active method based on piezoelectric elements introduced into a journal bearing system. The researchers examined an adaptive bearing that consisted of a mobile housing mounted on piezoelectric actuators. Four piezoelectric jacks made of lead zirconate titanate (PZT) or lead magnesium niobate (PMN) proved to yield strain ability

P. M. Przybylowicz $(\varangle) \cdot$ Z. Starczewski $\cdot$ P. Korczak-Komorowski

Warsaw University of Technology, Warsaw, Poland

E-mail: piotr.przybylowicz@simr.pw.edu.pl 
of order of the oil gap thickness under shaft loading of $30 \mathrm{~N}$ and operating frequency $8 \mathrm{kHz}$. Piezoelectric materials were also used by Przybyłowicz [18] who employed two contactless sensors which traced the current state (position and velocity) of the journal center. The signal was transformed and transmitted to a pair of two perpendicular piezoelectric jacks counteracting the current transverse movements of the rotor via direct driving of the bearing movable sleeve. A very interesting concept of stabilizing journal bearing systems was proposed by Kurnik [16]. The idea consisted in adding magnetic actuators to classical bearings. The actuators generated an attractive force that, when appropriately driven, placed the journal in regions within the oil gap characterized by safer operating conditions. It was shown that the stabilization efficiency had a maximum corresponding to more than a double increase in the critical rotation speed at which the system lost its stability.

Journal bearings are highly nonlinear systems. Their properties in terms of the inherent nonlinearity have been already investigated round the world since, at least, the seventies of the twentieth century. Holmes et al. [14] examined self-excited transverse vibration of a two-rotor four-bearing system subjected to misalignment of the supporting bearings. They presented theoretical considerations enabling one to obtain limit cycles which were in agreement with the vibration observed on large turbogenerators. A nonlinear response of a journal bearing being close to the loss of stability was discussed by Hollis and Taylor [13] who analytically determined the critical rotation speed using linear stability theory. They predicted that supercritical limit cycles can only exist within a narrow range of the Sommerfeld number, and in other cases, subcritical bifurcation should be expected. The results were also confirmed by numerical simulations. Brindley et al. [6] discussed the problem of free vibration of a rigid rotor supported by a short bearing oil film. The authors showed the existence of Hopf bifurcations at operating conditions close to the stability threshold and indicated that the presence of rotating unbalance may lead to even more complex dynamical behavior of the rotor. This indication was elaborated on by Brown et al. [7] who examined a simple model of a rigid rotor hydrodynamically supported by journal bearings. The researchers observed that the system starts to exhibit chaotic motion when the rotating unbalance exceeds the gravitational load. They made use of short bearing theory and found a fractal dimension of 2.15 in the obtained Poincaré plots. The required order of the unbalance was only an order of magnitude larger than the technologically acceptable level, thus achievable in the engineering practice. A symmetric rotor system with suitably selected ratio between the transverse and polar mass moments of inertia so as to avoid conical modes of vibration was studied by Adiletta et al. [2]. They confirmed numerically the onset of chaos for moderate values of the rotor unbalance. Chaotic dynamics of short oil film bearings was also discussed by Brown et al. [8].

In the recent past, incorporation of tilting-pad journal bearings to rotating machinery has increased because of their stabilizing influence on rotor-bearing systems. Yet in some operational regimes they may lead to subharmonic or even chaotic motion due to strongly nonlinear dynamics of such bearings [1]. Efforts to regulate the orbit of a rotating shaft supported on an active tilting-pad journal bearing were presented by Deckler et al. [11] who installed linear actuators with a feedback system controlling radial motion of the pads in real time.

Fundamental phenomena related to physics of flows in the lubricant itself have been investigated as well. The mixing in quasi-steady Stokes flows with slowly varying saddle stagnation points leading to large-scale chaos was analyzed by Kaper and Wiggins [15], whereas laminar mixing in two-dimensional time-periodic Stokes flows between eccentric cylinders was discussed by Galaktionov et al. [12].

Dynamic behavior of a rotor-bearing system consisting of a nonlinear support, linear damping, and a nonlinear elastic restoring force was investigated by Chang-Jian and Chen [9]. The authors assumed a turbulent lubricant flow model in the bearing and use the Runge-Kutta method for solving the differential equations of motion. They observed 2T-periodic, quasi-periodic and chaotic motions in the dynamical response of the system. In the last decade, researchers focused also on the problem of self-excited vibration of rotors supported by oil film bearings with the gyroscopic effect taken into account. Avramov and Borysiuk [4] considered an asymmetrical single-disk rotor exposed to hydrodynamical forces from journal bearings and observed that such interaction might lead to high-amplitude orbits of the rotor near criticality. Recently, Shi et al. [19] presented a sophisticated method based on Wolf and Rosenstein algorithms for detecting chaotic motion in rotor-bearing systems. The method incorporated phase space portraits together with the largest Lyapunov exponent and introduced a correlation integral function to trace the chaotic response accurately. The effect of application of nonlinear damping suspension to a rotor-bearing system in order to vibro-insulate the machine from environment was examined by Yan et al. [21]. It occurred that the nonlinearity of the suspension considerably influences the stability of the examined system depending on the kind of damping (linear, square or cubic) as it enlarges or reduces the range of stable operation of the rotor-bearing system. Depending on the bearing characteristics, Amamou and Chouchane [3] acknowledged that the stability threshold may occur either at a supercritical or at a subcritical Hopf bifurcation. Possible jumping from the equilibrium position of 
the rotor to a large limit cycle as well as the hysteresis phenomenon during variation of the rotor speed near criticality was confirmed in their study.

\section{Considered system}

A mechanical system whose main part is a rigid rotor of the entire mass $m$ spinning with an angular velocity $\omega$ is analyzed in this work. The rotor is asymmetrically loaded with an external transverse force $Q$ applied at a distance $a$ and $b$ from the left and right bearing, respectively. The rotor is supported on two journal bearings filled up with a lubricant. The plane model of the oil bearings is assumed. The bearing sleeves are not perfectly mounted to the rotor housing. They are attached to the rigid fundament through linearly viscoelastic elements characterized by the stiffnesses $k_{1}$ and $k_{2}$ for the left and right bearing as well as damping coefficients $c_{1}$ and $c_{2}$. The sleeves have masses $m_{1}$ and $m_{2}$, respectively. The entire length of the shaft is $a+b=L$. The system is shown in Fig. 1.

Additionally, the whole system is exposed to external kinematic excitation, i.e., motion of the fundament (floor) it is resting on. Such motion may be induced by operation of a neighboring rotating machine undergoing some unbalance. Forces transmitted through the common floor lead to some independent motion of the system under consideration. It can be treated as kind of a kinematic excitation applied to the rotor casing. In further analysis, it will be modeled as a quasi-static sinusoidal motion of amplitude $A$ and frequency $v$ applied synchronously to both supports.

It is worth mentioning at this moment that a similar problem of dynamics of a rotor mounted on hydrodynamic journal bearings subject to movement of the rigid supports due to seismic excitation was examined by Dakel et al. [10]. The authors proved that the rotor asymmetry and motions of the support generate timevarying parametric terms in the equations of motion which can lead to lateral dynamic instability. To support their conclusion, the researchers presented and discussed time history responses, orbits of the journal, Fourier transforms, bifurcation diagrams, and Poincaré maps.

Derive now equations of motion of the investigated system. Let the momentum and angular momentum laws be used for this purpose:

$$
\begin{aligned}
& \frac{\mathrm{d} \mathbf{B}}{\mathrm{d} t}=\mathbf{F}, \\
& \frac{\mathrm{d} \mathbf{K}_{C}}{\mathrm{~d} t}=\mathbf{M}_{C}
\end{aligned}
$$

where the momentum $\mathbf{B}$ is $\mathbf{B}=m \mathbf{v}_{C}=m\left[\dot{y}_{C}, \dot{z}_{C}\right]^{\mathrm{T}}$ and the angular momentum $\mathbf{K}_{C}=\mathbf{I}_{C} \boldsymbol{\omega}$, where $\mathbf{I}_{C}$ denotes the tensor of mass moments of inertia and $\omega$ the angular velocity vector. The coordinates describing the position of the gravity center of the rotor are shown in Fig. 2:

$$
y_{C}=y_{1}+a \frac{y_{2}-y_{1}}{a+b}, \quad z_{C}=z_{1}+a \frac{z_{2}-z_{1}}{a+b} .
$$

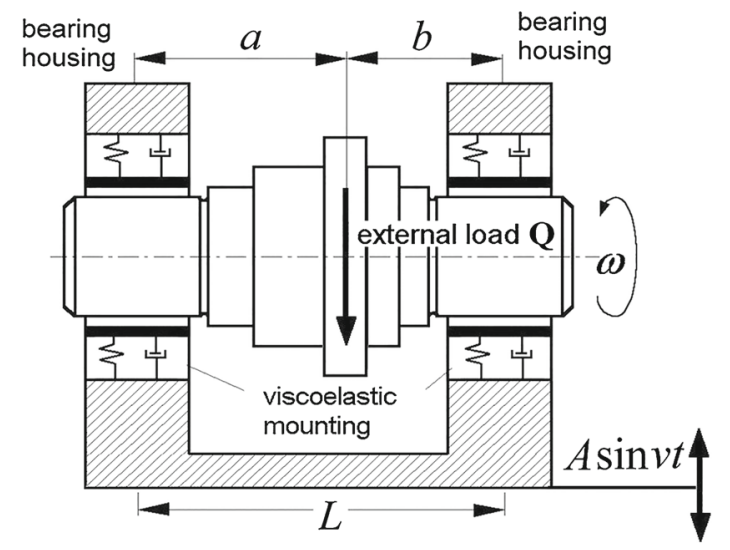

Fig. 1 The analyzed system: a rigid rotor supported on two oil film bearings with viscoelastically mounted sleeves 


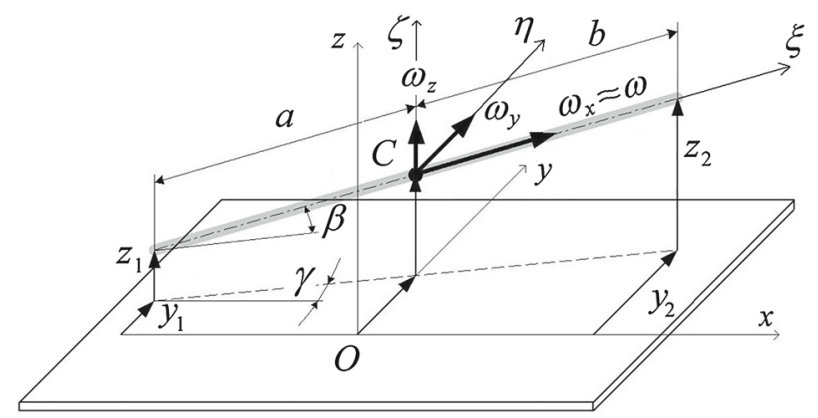

Fig. 2 Basic geometrical parameters describing the position of the rotor in arbitrary operational conditions

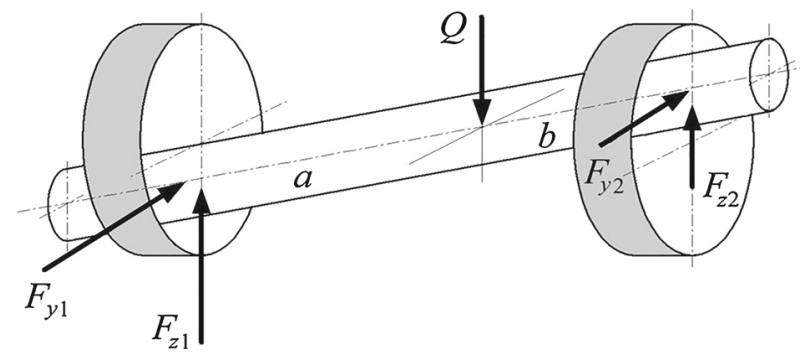

Fig. 3 Hydrodynamical and external forces acting on the rigid rotor

Introducing (2) into the Eq. (1.1), one obtains:

$$
\begin{aligned}
& \frac{m}{a+b}\left(\ddot{y}_{1} b+\ddot{y}_{2} a\right)=F_{y 1}+F_{y 2}, \\
& \frac{m}{a+b}\left(\ddot{z}_{1} b+\ddot{z}_{2} a\right)=F_{z 1}+F_{z 2}+Q
\end{aligned}
$$

where the forces $F_{y 1}, F_{z 1}, F_{y 2}, F_{z 2}$ are components of the hydrodynamic forces coming from the lubricant, see Fig. 3.

Let us find now an explicit relationship for the angular momentum making use of the angular velocity vector $\boldsymbol{\omega}$, which is, according to Fig. 2,

$$
\omega_{x} \approx \omega, \quad \omega_{y}=\frac{\dot{z}_{2}-\dot{z}_{1}}{a+b}+\omega \sin \gamma, \quad \omega_{z}=\frac{\dot{y}_{1}-\dot{y}_{2}}{a+b}+\omega \sin \beta
$$

where

$$
\sin \gamma=\frac{y_{2}-y_{1}}{a+b}, \quad \sin \beta=\frac{z_{2}-z_{1}}{a+b}
$$

and determining all elements of the inertia tensor $\mathbf{I}_{C}$ expressed in the moving coordinate system attached to the gravity center $C$ of the rotor with the axes parallel to the fixed coordinate system. Let $\xi, \eta, \zeta$ denote the principal axes of the rotor (see Fig. 2). By transforming the tensor $\mathbf{I}_{C}$ from the principal coordinate system $C \xi \eta \zeta$ into the system $C x y z$ via the known formula

$$
I_{k l}^{(x, y, x)}=\sum_{i=1}^{3} \sum_{j=1}^{3} I_{i j}^{(\xi, \eta, \zeta)} \cos \angle\left(\mathbf{e}_{k}^{(x, y, z)}, \mathbf{e}_{i}^{(\xi, \eta, \zeta)}\right) \cos \angle\left(\mathbf{e}_{l}^{(x, y, z)}, \mathbf{e}_{j}^{(\xi, \eta, \zeta)}\right)
$$

where $\mathbf{e}_{i}^{(x, y, z)}$ and $\mathbf{e}_{i}^{(\xi, \eta, \zeta)}, i=1,2,3$ are unit vectors of the axes in the $C \xi \eta \zeta$ and $C x y z$ systems, respectively, one finally gets:

$$
I_{x y} \approx\left(I_{2}-I_{1}\right) \sin \gamma, \quad I_{y z} \approx\left(I_{2}-I_{1}\right) \sin \beta
$$


where $I_{1}$ stands for the mass moment of inertia relative to the symmetry axis of the rotor, and $I_{2}$ for the moment relative to the other principal axes: $I_{1}=I_{11}^{(\xi, \eta, \zeta)}=I_{\xi \xi}=I_{\xi}, I_{2}=I_{22}^{(\xi, \eta, \zeta)}=I_{\eta \eta}=I_{\eta}$ and also $I_{2}=I_{33}^{(\xi, \eta, \zeta)}=I_{\zeta \zeta}=I_{\zeta}$. Having the above set forth, one concludes:

$$
\mathbf{K}_{C}=\mathbf{I}_{C} \boldsymbol{\omega}=\left(\begin{array}{ccc}
I_{x} & -I_{x y} & -I_{x z} \\
-I_{y x} & I_{y} & -I_{y z} \\
-I_{z x} & -I_{z y} & -I_{z}
\end{array}\right)\left(\begin{array}{c}
\omega_{x} \\
\omega_{y} \\
\omega_{z}
\end{array}\right)=\left(\begin{array}{c}
I_{1} \omega \\
I_{2} \frac{\dot{z}_{2}-\dot{z}_{1}}{L}-I_{1} \omega \frac{y_{2}-y_{1}}{L} \\
I_{2} \frac{\dot{y}_{1}-\dot{y}_{2}}{L}-I_{1} \omega \frac{z_{2}-z_{1}}{L}
\end{array}\right) .
$$

The above result (8) holds if the rate of lateral motion of the rotor is negligible with regard to the spin $\omega$, i.e., when $I_{y z} \omega_{y} \approx 0$ and $I_{y z} \omega_{z} \approx 0$. Differentiating $(8)$ with respect to time, one equals the result with the moment of forces as stated by the angular momentum law, see Eq. (1.2). This yields:

$$
\begin{aligned}
& \frac{I_{2}}{a+b}\left(\ddot{y}_{1}-\ddot{y}_{2}\right)+\omega \frac{I_{1}}{a+b}\left(\ddot{z}_{1}-\ddot{z}_{2}\right)=F_{y 1} a-F_{y 1} b, \\
& \frac{I_{2}}{a+b}\left(\ddot{z}_{2}-\ddot{z}_{1}\right)-\omega \frac{I_{1}}{a+b}\left(\ddot{y}_{2}-\ddot{y}_{1}\right)=-F_{z 1} a+F_{z 2} b,
\end{aligned}
$$

which, together with Eqs. (3), constitute the set of four governing equations of the rotor. Solving (3) and (9) with respect to accelerations $\ddot{y}_{1}, \ddot{z}_{1}, \ddot{y}_{2}$ and $\ddot{z}_{2}$, one finally arrives at:

$$
\begin{aligned}
& \ddot{y}_{1}=\frac{I_{1}}{I_{2}} \frac{a}{a+b} \omega\left(\dot{z}_{2}-\dot{z}_{1}\right)+\left(\frac{1}{m}+\frac{a^{2}}{I_{2}}\right) F_{y 1}+\left(\frac{1}{m}-\frac{a b}{I_{2}}\right) F_{y 2}, \\
& \ddot{z}_{1}=\frac{I_{1}}{I_{2}} \frac{a}{a+b} \omega\left(\dot{y}_{1}-\dot{y}_{2}\right)+\left(\frac{1}{m}+\frac{a^{2}}{I_{2}}\right) F_{z 1}+\left(\frac{1}{m}-\frac{a b}{I_{2}}\right) F_{z 2}+\frac{Q}{m} \frac{a}{a+b}, \\
& \ddot{y}_{2}=\frac{I_{1}}{I_{2}} \frac{b}{a+b} \omega\left(\dot{z}_{1}-\dot{z}_{2}\right)+\left(\frac{1}{m}-\frac{a b}{I_{2}}\right) F_{y 1}+\left(\frac{1}{m}+\frac{b^{2}}{I_{2}}\right) F_{y 2}, \\
& \ddot{z}_{2}=\frac{I_{1}}{I_{2}} \frac{b}{a+b} \omega\left(\dot{y}_{2}-\dot{y}_{1}\right)+\left(\frac{1}{m}-\frac{a b}{I_{2}}\right) F_{z 1}+\left(\frac{1}{m}+\frac{b^{2}}{I_{2}}\right) F_{z 2}+\frac{Q}{m} \frac{b}{a+b},
\end{aligned}
$$

The explicit forms of $F_{y 1}, F_{z 1}, F_{y 2}, F_{z 2}$ are derived in the following Section.

Obviously, the whole system has eight degrees of freedom. Elastically mounted bearing sleeves contribute to the entire system dynamics by introducing four additional dofs and kinematic excitation, see Fig. 4.

The corresponding equations of motion of the $i$-th sleeve in both transverse directions are:

$$
\begin{aligned}
m_{i} \ddot{y}_{s i}+c_{i} \dot{y}_{s i}+k_{i} y_{s i} & =-F_{y i}, \\
m_{i} \ddot{z}_{s i}+c_{i} \dot{z}_{s i}+k_{i} z_{s i} & =A\left(c_{i} v \cos v t+k_{i} \sin v t\right)-F_{z i} .
\end{aligned}
$$

$i=1,2$, where $y_{s i}$ and $z_{s i}$ are transverse displacements of the $i$-th sleeve in the $y$ and $z$ direction, respectively, $A$ is the amplitude of kinematic, excitation and $v$ is its frequency. Isotropic properties of the viscoelastic mounting of the sleeves are assumed: $c_{y i}=c_{z i}=c_{i}$ and $k_{y i}=k_{z i}=k_{i} . F_{y i}$ and $F_{z i}$ are of course hydrodynamic forces from the lubricant.

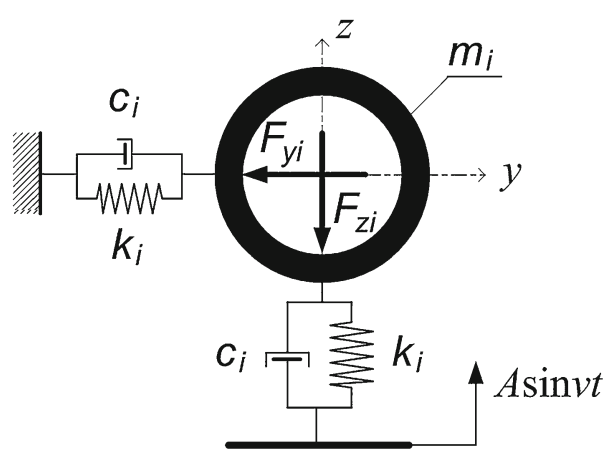

Fig. 4 A single journal bearing sleeve subject to sinusoidal kinematic excitation in the vertical direction $z$ 


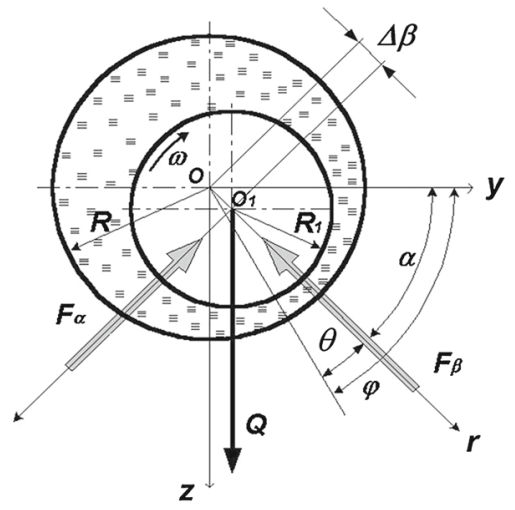

Fig. 5 Hydrodynamic uplift forces acting on the journal
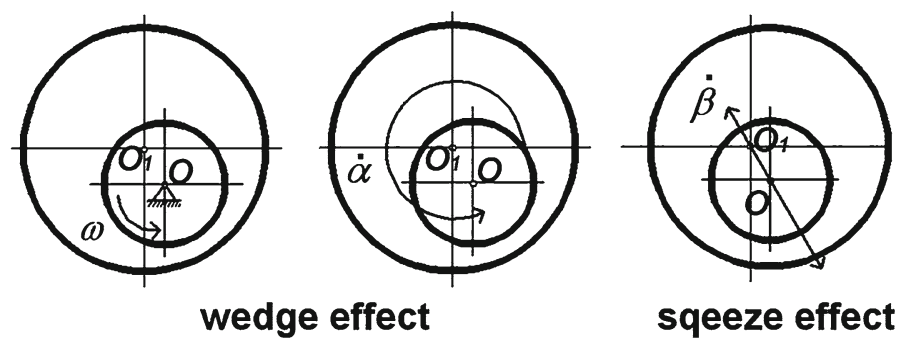

sqeeze effect

Fig. 6 Components of motion of the journal with respect to the bearing sleeve

\section{Hydrodynamic forces}

Assume journal bearings in which the axial flow of the lubricant is neglected with respect to the circumferential one. In such a case, hydrodynamic forces generated within the oil film can be explicitly determined by integrating the pressure field calculated with the aid of the simplified Reynolds equation written for the dynamic regime. Reynolds equation has the following form in the polar co-ordinates $\alpha-\beta$ for the plane (infinite length) bearing:

$$
\frac{\partial}{\partial \theta}\left[H^{3} \frac{\partial}{\partial \theta}\left(\frac{p}{\mu \delta^{2}}\right)\right]-6\left(\omega \frac{\partial H}{\partial \theta}+2 \frac{\partial H}{\partial t}\right)=0
$$

where $\theta$ is the current angular position with respect to circumferential is the position $\alpha$ of the journal $\theta=\varphi-\alpha$, see Fig. 5, $p$ is the oil pressure, $\mu$ is the dynamic viscosity of the lubricant, $R$ is the journal radius, $\Delta$ is the absolute bearing clearance, $\delta=\Delta / R$ is the relative oil gap, $H$ is the current thickness of the lubricating film: $H=\Delta(1+\beta \cos \theta), \beta$ is the relative eccentricity of the journal center, $\omega$ is the angular velocity of the journal, $t$ is time.

Neglecting the inertia forces of the lubricating medium and simplifying that the flow is isothermal, and then taking into consideration all components of the journal bearing motion, i.e., rotation around its own axis $(\omega)$, precession around the bearing axis $(\dot{\alpha})$ —corresponding to the wedge effect, and radial motion $(\dot{\beta})$ corresponding to the squeeze effect, see Fig. 6 , one can determine the hydrodynamic forces acting in the radial $(\beta)$ and circumferential $(\alpha)$ direction via double integration of (12).

For the $i$-th bearing, these forces are found to be [16,17]:

$$
\begin{aligned}
& F_{\beta i}=-\frac{12 \mu_{i} R_{i} L_{i}}{\Delta_{i}^{2}}\left[\frac{\beta_{i}^{2}\left(\omega-2 \dot{\alpha}_{i}\right)}{\left(1-\beta_{i}^{2}\right)\left(2+\beta_{i}^{2}\right)}+\frac{\beta_{i} \dot{\beta}_{i}}{1-\beta_{i}^{2}}+\frac{2 \dot{\beta}_{i}}{\sqrt{\left(1-\beta_{i}^{2}\right)^{3}}} \operatorname{arctg} \sqrt{\frac{1+\beta_{i}}{1-\beta_{i}}}\right], \\
& F_{\alpha i}=\frac{6 \pi \mu_{i} R_{i} L_{i}}{\Delta_{i}^{2}} \frac{\beta_{i}\left(\omega-2 \dot{\alpha}_{i}\right)}{\sqrt{\left(1-\beta_{i}^{2}\right)\left(2+\beta_{i}^{2}\right)}}
\end{aligned}
$$

where $L_{i}$ denotes the length of the $i$ th journal. Equation (13) is valid provided that the oil film exists in the region $(0, \pi)$ for the wedge effect, and in $(-\pi / 2, \pi / 2)$ for the squeeze one. Such restrictions are known 
as Gümbel's boundary conditions. For further convenience, the above expressions are then transformed into relationships described in the Cartesian coordinates. Having already introduced the horizontal and vertical displacement of the $i$ th bearing $y_{i}$ and $z_{i}$, respectively, which are related with the polar coordinates by the formula:

$$
\beta_{i}=\frac{1}{\Delta_{i}} \sqrt{Y_{i}^{2}+Z_{i}^{2}} \text { and } \alpha_{i}=\arctan \frac{Z_{i}}{Y_{i}},
$$

one obtains:

$$
\begin{aligned}
F_{\alpha i}\left(Y_{i}, \dot{Y}_{i}, Z_{i}, \dot{Z}_{i}\right)= & \frac{6 \pi \mu R^{3} L\left[\omega\left(Y_{i}^{2}+Z_{i}^{2}\right)+2\left(\dot{Y}_{i} Z_{i}-Y_{i} \dot{Z}_{i}\right)\right]}{\left(2 \Delta^{2}+Y_{i}^{2}+Z_{i}^{2}\right) \sqrt{Y_{i}^{2}+Z_{i}^{2}} \sqrt{\Delta^{2}-Y_{i}^{2}-Z_{i}^{2}}}, \\
F_{\beta i}\left(Y_{i}, \dot{Y}_{i}, Z_{i}, \dot{Z}_{i}\right)= & -12 \pi \mu R^{3} L\left\{\frac{Y_{i} \dot{Y}_{i}+Z_{i} \dot{Z}_{i}}{\Delta^{2}\left(\Delta^{2}-Y_{i}^{2}-z_{i}^{2}\right)}\right. \\
& -\frac{\omega\left(Y_{i}^{2}+Z_{i}^{2}\right)-2\left(\dot{Y}_{i} Z_{i}-Y_{i} \dot{Z}_{i}\right)}{\left(2 \Delta^{2}+Y_{i}^{2}+Z_{i}^{2}\right)\left(\Delta^{2}-Y_{i}^{2}-Z_{i}^{2}\right)} \\
& \left.+2 \frac{Y_{i} \dot{Y}_{i}+Z_{i} \dot{Z}_{i}}{\sqrt{Y_{i}^{2}+Z_{i}^{2}} \sqrt{\left(\Delta^{2}-Y_{i}^{2}-Z_{i}^{2}\right)^{3}}} \arctan \sqrt{\frac{\Delta+\sqrt{Y_{i}^{2}+Z_{i}^{2}}}{\Delta-\sqrt{Y_{i}^{2}+Z_{i}^{2}}}}\right\}
\end{aligned}
$$

where, additionally, it has been simplified that all geometrical and material parameters of both bearings (clearances, lengths, radii, oil viscosities) are the same. In the above equations, $Y_{i}$ and $Z_{i}$ are relative displacements of the journal centers:

$$
Y_{i}=y_{i}-y_{s i}, \quad Z_{i}=z_{i}-z_{s i}
$$

while $y_{i}$ and $z_{i}$ are absolute displacements of the journal centers, $y_{s i}$, and $z_{i s}$, of the sleeves. At the end, let us finally write down the expressions for forces appearing in the equations of motion (10). They are as follows:

$$
\begin{aligned}
F_{y i} & =F_{\beta i} \cos \alpha_{i}-F_{\alpha i} \sin \alpha_{i}, \\
F_{z i} & =F_{\beta i} \sin \alpha_{i}+F_{\alpha i} \cos \alpha_{i}
\end{aligned}
$$

where

$$
\sin \alpha_{i}=\frac{z_{i}}{\sqrt{y_{i}^{2}+z_{i}^{2}}}, \quad \cos \alpha_{i}=\frac{y_{i}}{\sqrt{y_{i}^{2}+z_{i}^{2}}} .
$$

Now the complete system of eight equations of motion-four (10) with supporting (14-19) and the other four (11) containing kinematic excitation- has been determined. It is a basis for further numerical exploration.

\section{Results of numerical analysis}

The derived equations of motion are strongly coupled, highly nonlinear and very large in volume. To the best of the authors' knowledge, an exact analytical solution to them probably does not exist at all; however, known are analytical approximations describing near-critical behavior found from Hopf's bifurcation theory. In this paper, only a numerical study has been carried out by making direct use of the Runge-Kutta method of the fourth order. The main aim has been to determine bifurcation diagrams of transverse displacements of the journal bearing centers of both rotor ends as functions of the applied amplitude of kinematic excitation. Bifurcation diagrams are the easiest way to distinguish between regions of regular (periodic) and irregular (including chaotic) response of the system. The amplitude variation ranged from 0 up to $8 \times 10^{-4} \mathrm{~m}$. It has been assumed that the kinematic excitation comes from a similar machine operating within similar conditions (e.g., a twin generator) and goes through a common fundament to the rotor system considered. The excitation frequency has been kept constant at $v=360 \mathrm{rad} / \mathrm{s}$, which corresponds to the angular velocity of the analyzed system. Three cases of the bearing sleeves mounting the rotor housing have been taken into account-very stiff (which could be regarded as almost perfectly rigid) having the elasticity coefficient of $k=10^{10} \mathrm{~N} / \mathrm{m}$, 

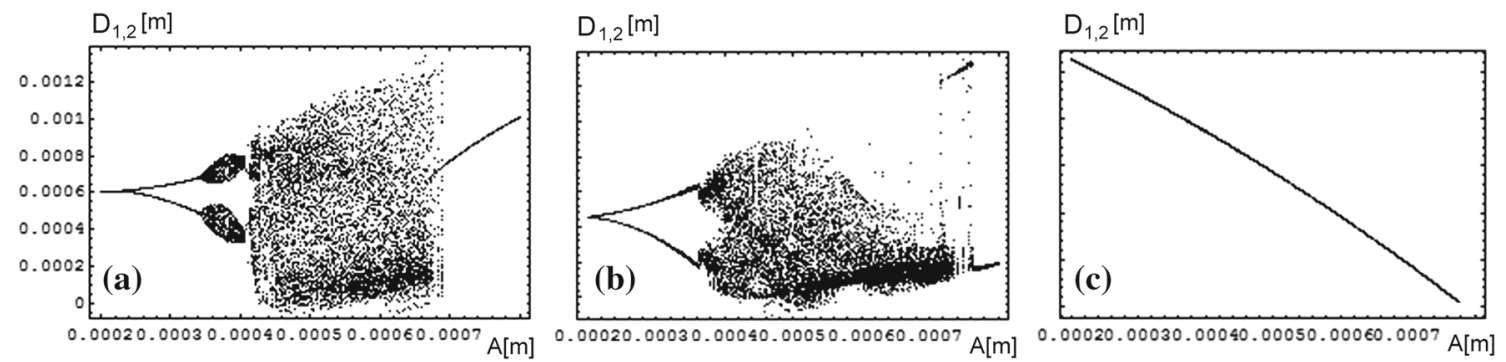

Fig. 7 Bifurcation diagram of vibration amplitudes of both bearings for a fully symmetric system $(a=b=0.5 l)$ and different stiffnesses of the elastic mounting of the sleeves: $\mathbf{a} k_{1}=k_{2}=10^{10} \mathrm{~N} / \mathrm{m}, \mathbf{b} k_{1}=k_{2}=10^{8} \mathrm{~N} / \mathrm{m}, \mathbf{c} k_{1}=k_{2}=10^{6} \mathrm{~N} / \mathrm{m}$
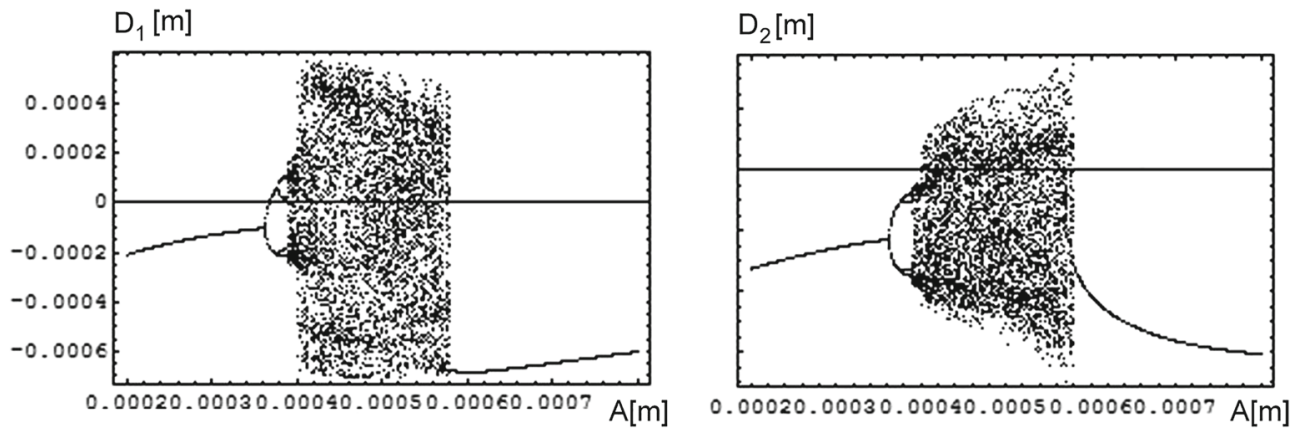

Fig. 8 Bifurcation diagram of vibration amplitudes of both bearings (1 - left bearing, 2-right one) for a strongly asymmetric system $(a=0.2 l, b=0.8 l)$ and high stiffnesses of the elastic mounting $k_{1}=k_{2}=10^{10} \mathrm{~N} / \mathrm{m}$

moderate (intermediate) with $k=10^{8} \mathrm{~N} / \mathrm{m}$, and quite soft with $k=10^{6} \mathrm{~N} / \mathrm{m}$. The degree of asymmetry varied from zero $(a=b=0.5 l)$ up to strong deviation of the point of application of transverse load by $90 \%$ $(a=0.2 l, b=0.8 l)$. Apart from bifurcation diagrams, Poincaré sections and, in some cases, phase portraits together with Fourier spectra have been determined. The values of main parameters of the rotor-bearing system used in the simulations are the following: rotor length $l=a+b=1 \mathrm{~m}$, journal radius $R=0.1 \mathrm{~m}$, journal length $L=0.1 \mathrm{~m}$, bearing clearance $\Delta=1 \times 10^{-5} \mathrm{~m}$, absolute viscosity of the lubricant $\mu=0.319 \mathrm{Ns} / \mathrm{m}^{2}$, angular velocity of the rotor $\omega=360 \mathrm{rad} / \mathrm{s}$.

In the following Figures, $D$ stands for the displacement of the given journal center: $D_{i}=\sqrt{y_{i}^{2}+z_{i}^{2}}, V$ stands for the velocity in the respective direction: $V_{i}=\sqrt{\dot{y}_{i}^{2}+\dot{z}_{i}^{2}}, i=1,2$.

In Fig. 7, bifurcation diagrams for a fully symmetric system are presented. It is clearly seen that almost perfect mounting of the bearing sleeves leads to chaotic vibration of the rotor within a certain region of the excitation amplitude (from $4 \times 10^{-4}$ to $7 \times 10^{-4} \mathrm{~m}$ ). Evidently seen is the area of smooth oscillatory behavior of the rotor as equally clear are the areas in which the system undergoes sudden bifurcations resulting in chaotic response. Such a situation preserves until the excitation amplitude exceeds another threshold above which the dynamics becomes smooth again. More elastic mounting slightly enlarges the chaotic zone toward larger amplitudes, but deteriorates the irregularity at the end of it. Yet it occurs that soft mounting of the sleeves eliminates chaos completely. A similar scenario is observed in both bearings for a strongly asymmetric system, see Figs. 8, 9 and 10.

The effect of stiffness in the bearing sleeve-rotor housing connection on kind of the dynamic response of the symmetric system is presented in Figs. 11, 12 and 13. Three types of diagrams are shown in that case: phase trajectories (Fig. 11), Fourier spectra (Fig. 12), and Poincaré maps corresponding to the above trajectories (Fig. 13).

In Fig. 14, Poincaré sections are the presented for some selected (increasing) amplitudes of the excitation amplitude $A=3 \times 10^{-4}, 5 \times 10^{-4}$ and $7 \times 10^{-4} \mathrm{~m}$ for a weakly asymmetric system and an almost perfectly rigid mounting of the bearing sleeves to the rotor housing.

Figure 15 depicts maps for both the left and right bearing in the same conditions as in Fig. 14 but with more elastic mounting of the sleeve (by two orders of magnitude). As can be seen, irregular motion stretches over 


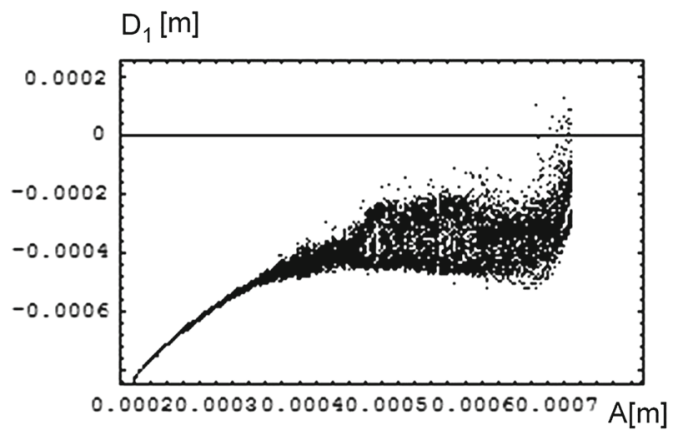

\section{$\mathrm{D}_{2}[\mathrm{~m}]$}

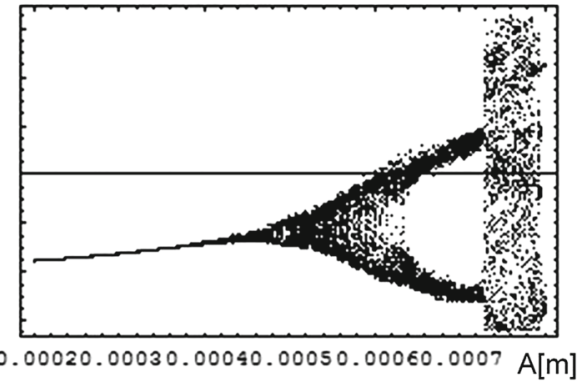

Fig. 9 Bifurcation diagram of vibration amplitudes of both bearings (1-left bearing, 2-right one) for a strongly asymmetric system $(a=0.2 l, b=0.8 l)$ and moderate stiffnesses of the elastic mounting $k_{1}=k_{2}=10^{8} \mathrm{~N} / \mathrm{m}$
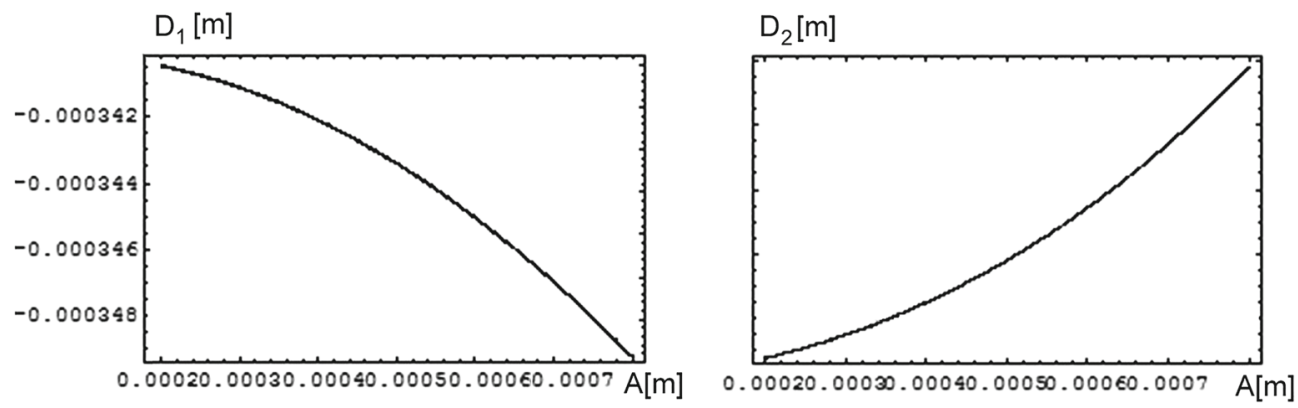

Fig. 10 Bifurcation diagram of vibration amplitudes of both bearings (1-left bearing, 2-right one) for a strongly asymmetric system $(a=0.2 l, b=0.8 l)$ and low stiffnesses of the elastic mounting $k_{1}=k_{2}=10^{6} \mathrm{~N} / \mathrm{m}$
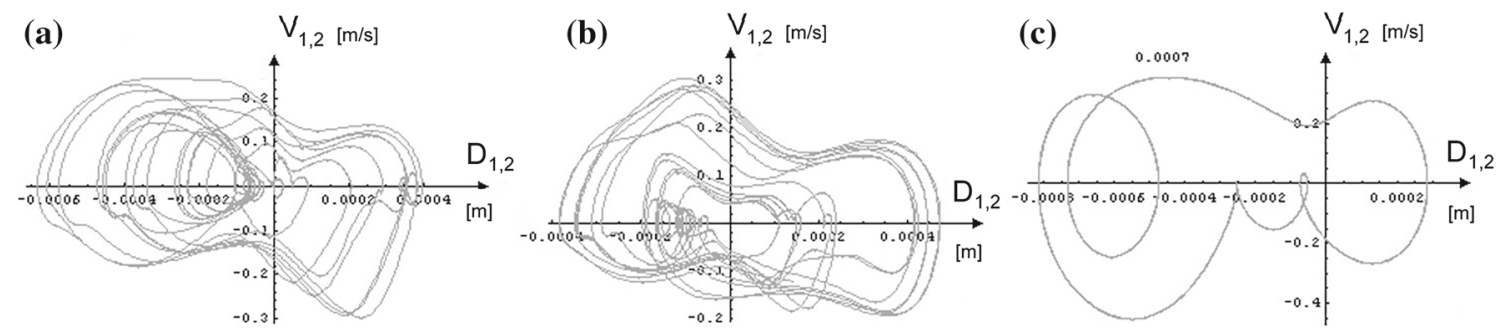

Fig. 11 Phase trajectories of both journal centers for a fully symmetric system $(a=b=0.5 l)$ and different stiffnesses of the elastic mounting of the sleeves: a $k_{1}=k_{2}=10^{10} \mathrm{~N} / \mathrm{m}, \mathbf{b} k_{1}=k_{2}=10^{8} \mathrm{~N} / \mathrm{m}, \mathbf{c} k_{1}=k_{2}=10^{6} \mathrm{~N} / \mathrm{m}$. Amplitude of kinematic excitation: $A=5 \times 10^{-4} \mathrm{~m}$, angular frequency $v=360 \mathrm{rad} / \mathrm{s}$
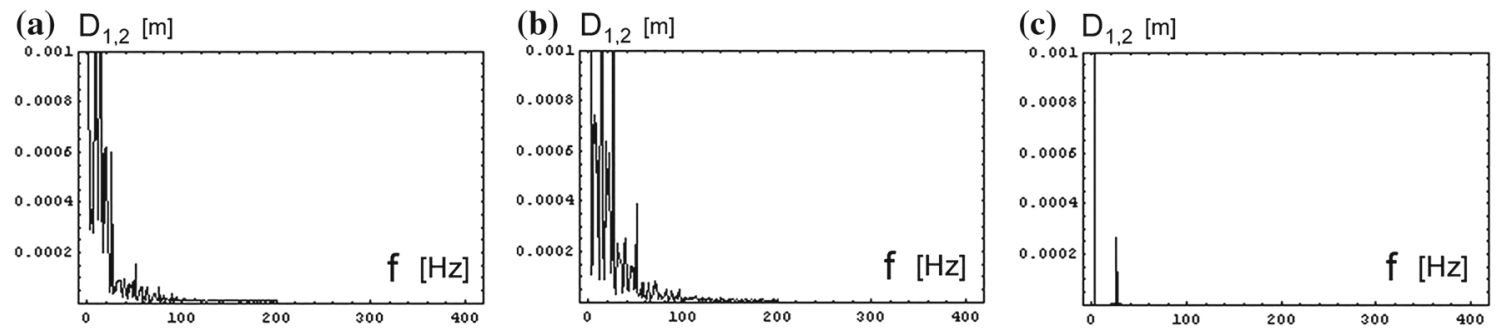

Fig. 12 Fouriers spectra of the vibration amplitude of both journal centers for a fully symmetric system and different stiffnesses of the elastic mounting of the sleeves: $\mathbf{a} k_{1}=k_{2}=10^{10} \mathrm{~N} / \mathrm{m}, \mathbf{b} k_{1}=k_{2}=10^{8} \mathrm{~N} / \mathrm{m}, \mathbf{c} k_{1}=k_{2}=10^{6} \mathrm{~N} / \mathrm{m}$. Amplitude of kinematic excitation: $A=5 \times 10^{-4} \mathrm{~m}$, angular frequency $v=360 \mathrm{rad} / \mathrm{s}$ 


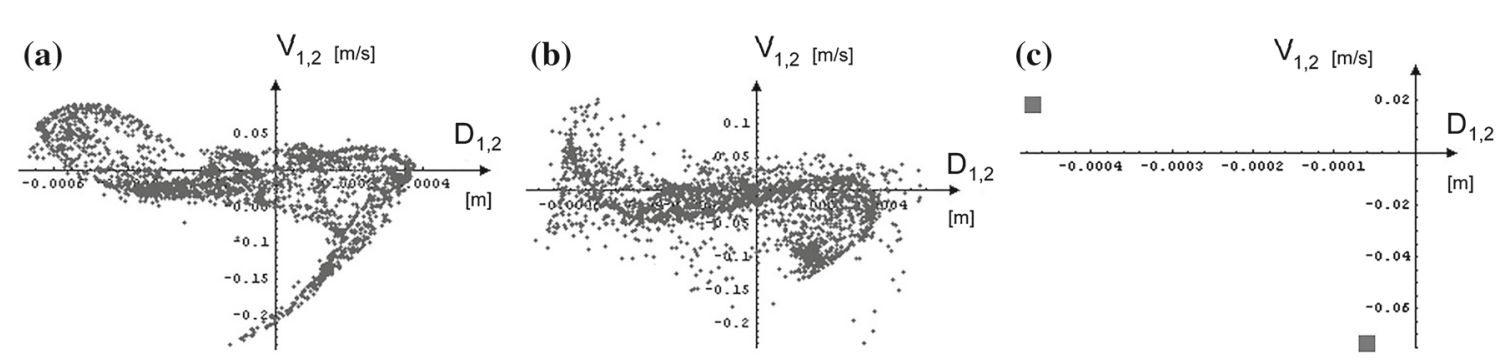

Fig. 13 Poincare maps of motion of both journal centers for a fully symmetric system and different stiffnesses of the elastic mounting of the sleeves: $\mathbf{a} k_{1}=k_{2}=10^{10} \mathrm{~N} / \mathrm{m}, \mathbf{b} k_{1}=k_{2}=10^{8} \mathrm{~N} / \mathrm{m}, \mathbf{c} k_{1}=k_{2}=10^{6} \mathrm{~N} / \mathrm{m}$. Amplitude of kinematic excitation: $A=5 \times 10^{-4} \mathrm{~m}$, angular frequency $v=360 \mathrm{rad} / \mathrm{s}$

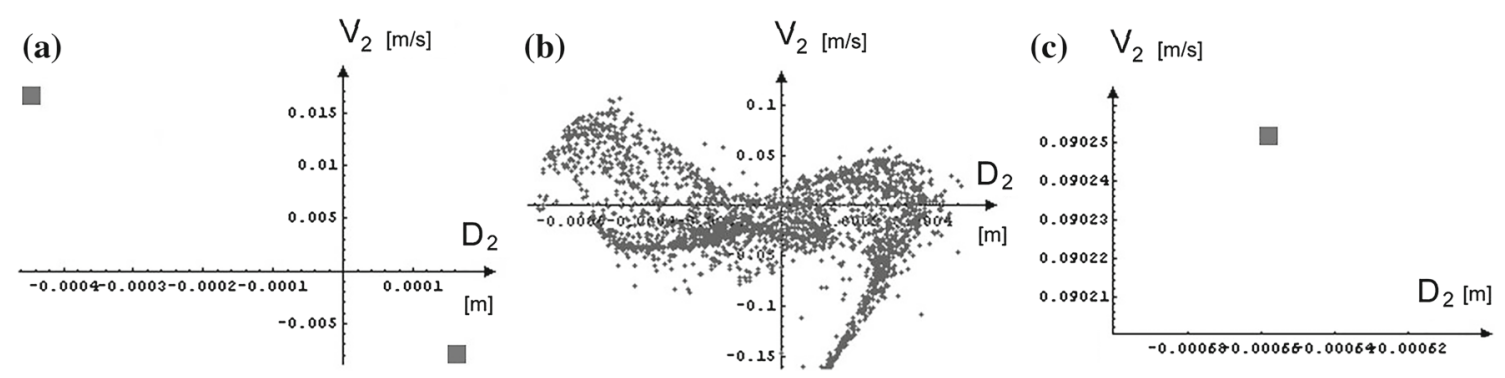

Fig. 14 Poincare maps for trajectories of the right bearing for various amplitudes of kinematic excitation: $\mathbf{a} A=3 \times 10^{-4} \mathrm{~m}$, b $A=5 \times 10^{-4} \mathrm{~m}, \mathbf{c} A=7 \times 10^{-4} \mathrm{~m}$. Very rigid mounting of the sleeve $\left(k_{2}=10^{10} \mathrm{~N} / \mathrm{m}\right)$ and weak asymmetry $(a=0.4 l, b=0.6 l)$
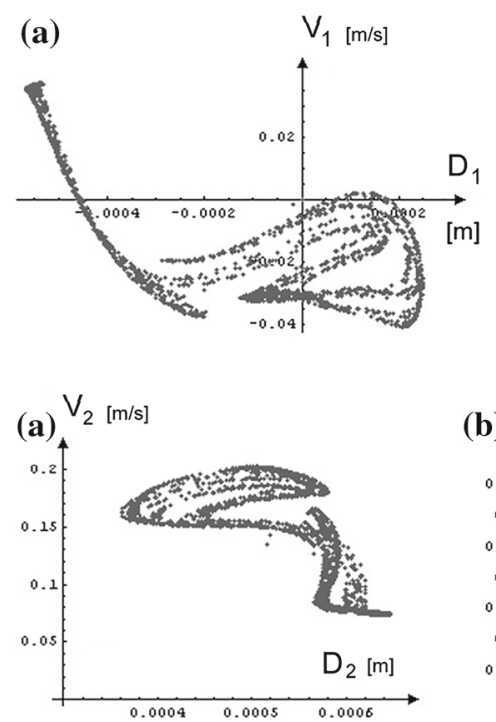

(b)

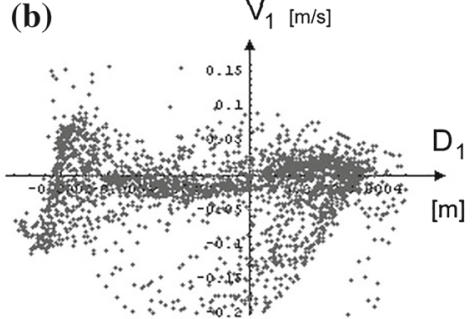

(b) $V_{2}[\mathrm{~m} / \mathrm{s}]$

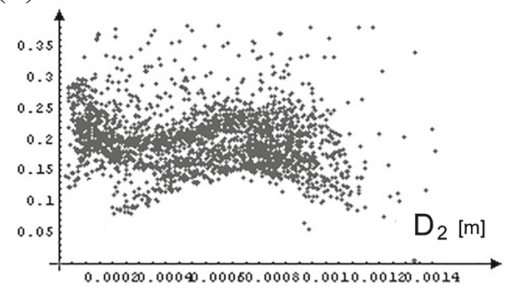

(c)

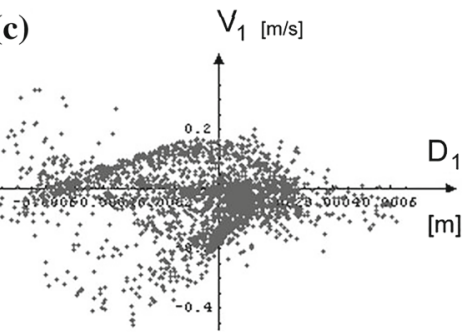

(c) $V_{2[\mathrm{~m} / \mathrm{s}]}$

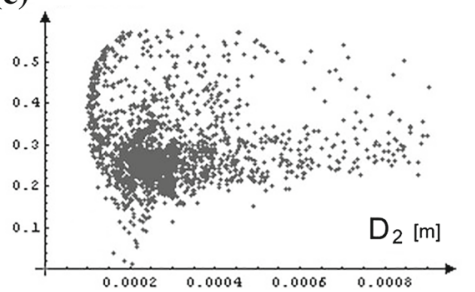

Fig. 15 Poincare maps for trajectories of the left bearing (upper row) and right bearing (lower row) for various amplitudes of kinematic excitation: a $A=3 \times 10^{-4} \mathrm{~m}, \mathbf{b} A=5 \times 10^{-4} \mathrm{~m}, \mathbf{c} A=7 \times 10^{-4} \mathrm{~m}$. Moderately stiff mounting of the sleeve $\left(k_{1}=k_{2}=10^{8} \mathrm{~N} / \mathrm{m}\right)$ and weak asymmetry $(a=0.4 l, b=0.6 l)$

the entire analyzed region of the amplitude variation. At $A=3 \times 10^{-4}$, structures resembling typical strange attractors are noticeable (Fig. 15a), which may be an indirect prove of the presence of chaos in the system.

As expected, low stiffness of the sleeve-housing joining element brings no aperiodic response of the rotor and no matter the system is weakly asymmetrical (Fig. 16) or strongly asymmetrical (Fig. 17), see the small squares in the Poincaré maps (one-periodic vibration).

But again, almost perfect attachment of the journal bearings and their sleeves to the machine housing may lead to irregular (possibly chaotic) motion of the system, see Fig. 18b. 


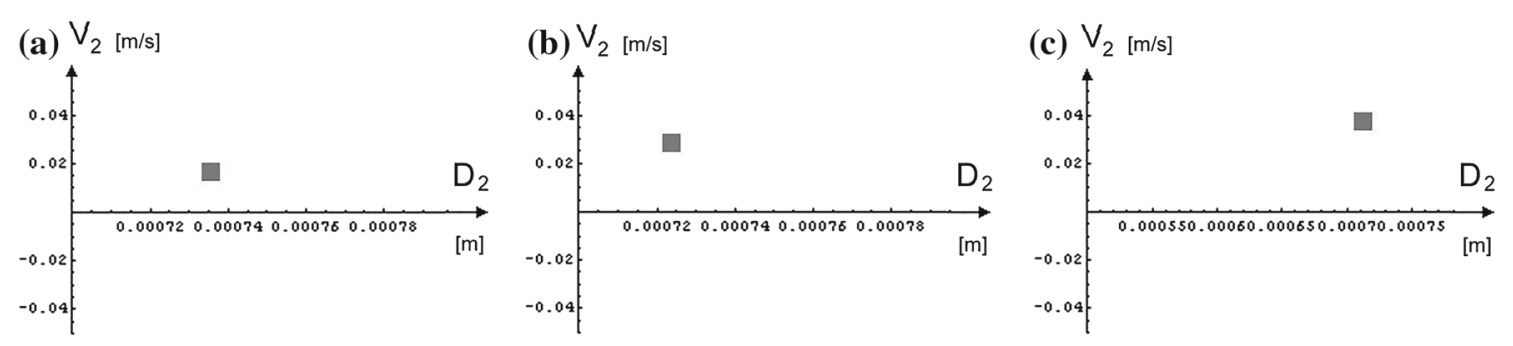

Fig. 16 Poincare maps for trajectories of the right bearing for various amplitudes of kinematic excitation: $\mathbf{a} A=3 \times 10^{-4} \mathrm{~m}$, b $A=5 \times 10^{-4} \mathrm{~m}, \mathbf{c} A=7 \times 10^{-4} \mathrm{~m}$. Elastic mounting of the sleeve $\left(k_{2}=10^{6} \mathrm{~N} / \mathrm{m}\right)$ and weak asymmetry $(a=0.4 l, b=0.6 l)$
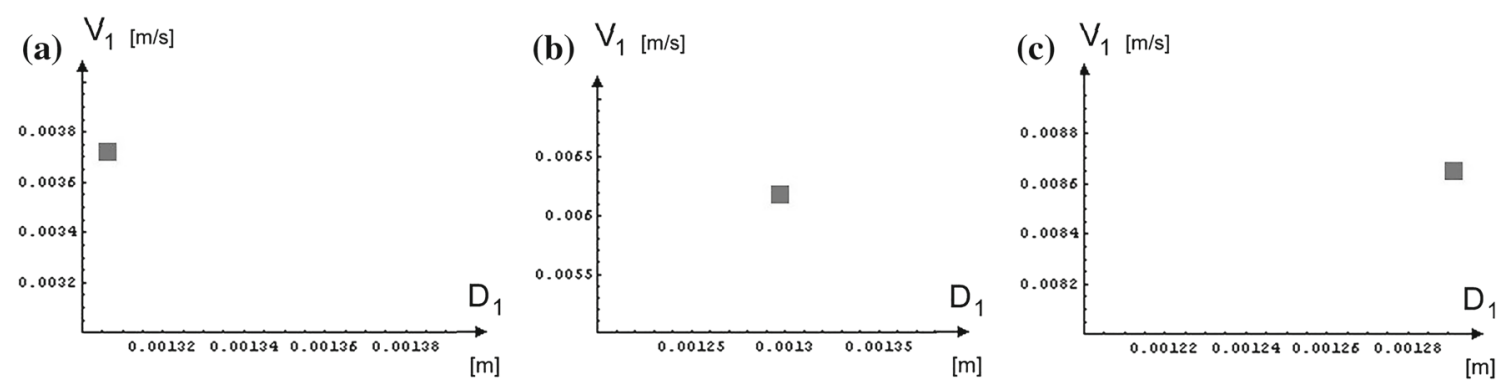

Fig. 17 Poincare maps for trajectories of the left bearing for various amplitudes of kinematic excitation: $\mathbf{a} A=3 \times 10^{-4} \mathrm{~m}$, b $A=5 \times 10^{-4} \mathrm{~m}, \mathbf{c} A=7 \times 10^{-4} \mathrm{~m}$. Soft mounting of the sleeve $\left(k_{1}=10^{6} \mathrm{~N} / \mathrm{m}\right)$ and strong asymmetry $(a=0.2 l, b=0.8 l)$
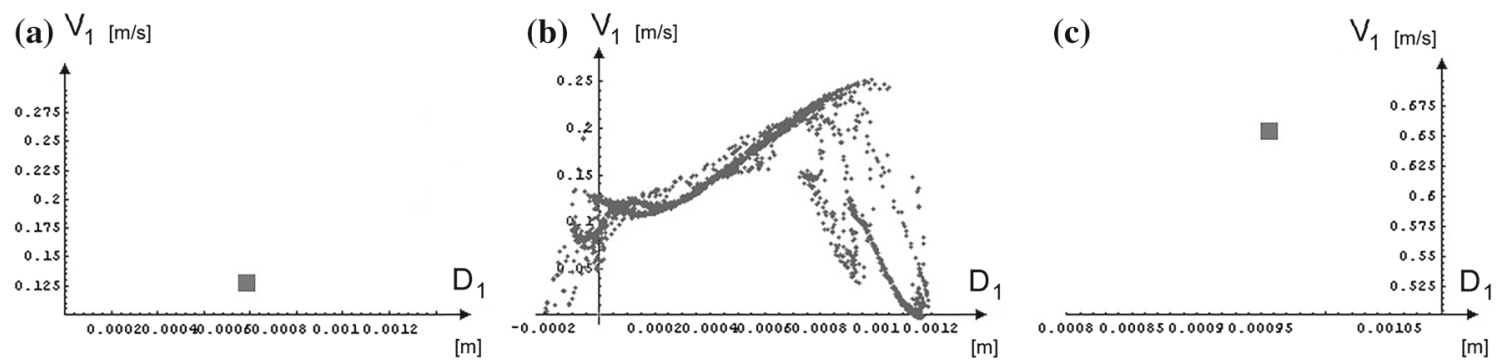

Fig. 18 Poincare maps for trajectories of the left bearing for various amplitudes of kinematic excitation: $\mathbf{a} A=3 \times 10^{-4} \mathrm{~m}$, b $A=5 \times 10^{-4} \mathrm{~m}, \mathbf{c} A=7 \times 10^{-4} \mathrm{~m}$. Very stiff mounting of the sleeve $\left(k_{1}=10^{10} \mathrm{~N} / \mathrm{m}\right)$ and strong asymmetry $(a=0.2 l, b=0.8 l)$

\section{Concluding remarks}

The carried out numerical analysis has confirmed that asymmetrically loaded rigid rotors supported on journal bearings with flexibly mounted sleeves may exhibit irregular or even chaotic vibration when exposed to kinematic excitation from the foundation. Such motion may be brought about by independent operation of a neighboring rotating machine. The character of the observed chaos is purely deterministic and comes from the nonlinearity of the considered system. Since all parameters defining the analyzed model are certain (nonprobabilistic), no Wiener chaos expansion, i.e., polynomial chaos, is expected in the system [20].

Two important parameters are especially vital for the rotor system to exhibit chaotic dynamics: the amplitude of the excitation and the rigidity of the elastically mounted bearing sleeves. Crucial is the degree of asymmetry of the applied load as well; however, the entire system is strongly sensitive to those two parameters: excitation amplitude and mounting rigidity. The presence of damping in the sleeve joint is of minor importance. In this study, it has been determined that more elastic connection between the sleeves and bearing housing may entirely eliminate chaotic response within the whole region of variation of the excitation amplitude. It is then possible, by appropriate selection of elasticity characteristics, to protect the rotor-bearing system from strange and hazardous dynamical behavior already at the design stage. Obviously, it is only a passive approach to the problem of chaos and cannot be understood in terms of control of any kind at all. The use of elastic rings with too soft characteristic may prevent the rotor from irregular motion, but may occur inadvisable or even not allowable because of purely operational reasons (poor load capacity, excessive resonance in transient states, 
etc.). Further research in this direction together with sensitivity studies is required. Another problem still to be addressed is the appearance of irregular motions due to the rotor itself, i.e., coming from natural unbalance of the system. Although the source of the disturbance may look similar to kinematic excitation, the results found so far are not applicable to such a case.

Acknowledgments The authors would like to acknowledge financial support given by scientific Project No. N N501 133738 granted by Polish Ministry of Science and Higher Education.

Open Access This article is distributed under the terms of the Creative Commons Attribution 4.0 International License (http:// creativecommons.org/licenses/by/4.0/), which permits unrestricted use, distribution, and reproduction in any medium, provided you give appropriate credit to the original author(s) and the source, provide a link to the Creative Commons license, and indicate if changes were made.

\section{References}

1. Abu-Mahfouz, I., Adams, M.L.: Numerical study of some nonlinear dynamics of a rotor supported on a three-pad tilting pad journal bearing. ASME Trans. J. Vibr. Acoust. 127, 262-272 (2005)

2. Adiletta, G., Guido, A.R., Rossi, C.: Chaotic motions of a rigid rotor in short journal bearings. Nonlinear Dyn. 10, 251269 (1996)

3. Amamou, A., Chouchane, M.: Nonlinear stability analysis of long hydrodynamic journal bearings using numerical continuation. Mech. Mach. Theory 72, 17-24 (2014)

4. Avramov, K.V., Borysiuk, O.V.: Nonlinear dynamics of one disk asymmetrical rotor supported by two journal bearings. Nonlinear Dyn. 67, 1201-1219 (2012)

5. Bonneau, O., Lecoutre, E., Frenê, J.: Dynamic behavior of a rigid shaft mounted in an active bearing. In: Muszynska, A. (eds.) Proceedings of ISROMAC-7, The 7-th International Symposium on Transport Phenomena and Dynamics of Rotating Machinery, 22-26 Feb. 1998, Honolulu, HI, Vol. A, 30-37 (1998)

6. Brindley, J., Savage, M.D., Taylor, C.M.: The nonlinear dynamics of journal bearings. Philos. Trans. R. Soc. Lond. Ser. A Math. Phys. Sci. 332, 107-119 (1990)

7. Brown, R.D., Addison, P., Chan, A.H.C.: Chaos in the unbalance response of journal bearings. Nonlinear Dyn. 5, 421$432(1994)$

8. Brown, R.D., Drummond, G., Addison, P.S.: Chaotic response of a short journal bearing. Proc. Inst. Mech. Eng. Part J J. Eng. Tribol. 214, 387-400 (2000)

9. Chang-Jian, C.-W., Chen, C.-K.: Bifurcation and chaos analysis of a flexible rotor supported by turbulent long journal bearings. Chaos, Solitons, Fract. 34, 1160-1179 (2007)

10. Dakel, M.Z., Baguet, S., Dufour, R.: Nonlinear dynamics of a support-excited flexible rotor with hydrodynamic journal bearings. J. Sound Vibr. 333, 2774-2799 (2014)

11. Deckler, D.C., Veillette, R.J., Braun, M.J.: Simulation and control of an active tilting-pad journal bearing. Tribol. Trans. 47, 440-458 (2004)

12. Galaktionov, O.S., Anderson, P.D., Peters, G.W.M.: Structure development during chaotic mixing in the journal bearing flow. Phys. Fluids 14, 3009-3017 (2002)

13. Hollis, P., Taylor, D.L.: Hopf bifurcation to limit cycles in fluid film bearings. J. Tribol. 108, 184-189 (1986)

14. Holmes, A.G., McC Ettles, C.M., Mayes, I.W.: The dynamics of multi-rotor systems supported on oil film bearings. J. Mech. Des. 100, 156-164 (1978)

15. Kaper, T., Wiggins, S.: An analytical study of transport in Stokes flows exhibiting large-scale chaos in the eccentric journal bearing. J. Fluid Mech. 253, 211-243 (1993)

16. Kurnik, W.: Magnetic stabilization of a rotor with hydrodynamic bearings. Mach. Dyn. Prob. 7, 117-133 (1994)

17. Pinkus, O., Sternlicht, B.: Theory of hydrodynamic lubrication. Mc-Graw Hill, New York (1961)

18. Przybyłowicz, P.M.: Active stabilisation of a rigid rotor by piezoelectrically controlled mobile journal bearing system. Aust. J. Mech. Eng. 1, 123-127 (2004)

19. Shi, M.-L., Wang, D.-Z., Zhang, J.-G.: An improved method of detecting chaotic motion for rotor-bearing systems. J. Shanghai Jiaotong Univ. (Sci.) 18, 229-236 (2013)

20. Wiener, N.: The homogeneous chaos. Am. J. Math. 60, 897-936 (1938)

21. Yan, S., Dowell, E.H., Lin, B.: Effects of nonlinear damping suspension on nonperiodic motions of a flexible rotor in journal bearings. Nonlinear Dyn. 78, 1435-1450 (2014) 